\title{
CUANTIFICACIÓN DE METALES EN AGUAS DE RIEGO. ESTUDIO DE CASO EN LA PROVINCIA DE CHIMBORAZO
}

\author{
Metal Quantification in Irrigation Waters. \\ A Case Study in The Province of Chimborazo
}

\author{
${ }^{1}$ J. Idrovo*, ${ }^{1}$ I. Gavilanes, ${ }^{1}$ N. Veloz, ${ }^{2}$ R. Erazo, ${ }^{3} \mathrm{H}$. Valverde
}

${ }^{1}$ Facultad de Ciencias, Escuela Superior Politécnica de Chimborazo, EC060155-Riobamba (Chimborazo), Ecuador

${ }^{2}$ Centro de Servicios Técnicos y Transferencia Tecnológica Ambiental, Escuela Superior Politécnica de Chimborazo, EC 060155-Riobamba (Chimborazo), Ecuador

${ }^{3}$ Unidad de Admisión y Nivelación, Escuela Superior Politécnica de Chimborazo,

EC060155-Riobamba (Chimborazo), Ecuador

*e-mail: j.idrovo@espoch.edu.ec

\section{$R$ esumen}

El crecimiento de la agroindustria en el Ecuador ha causado en los últimos años graves problemas de contaminación ambiental que afectan a sus recursos naturales, por lo cual es importante aplicar estrategias cada vez más eficaces para determinar las fuentes de contaminación y mitigar sus impactos en el ambiente. Una de las más importantes es la presencia de metales pesados, por lo tanto, es fundamental la determinación cuantitativa de ellos para llevar a cabo su gestión adecuada. La provincia de Chimborazo se encuentra ubicada en la zona central de los Andes ecuatorianos y una de sus principales agroindustrias es la relacionada con la producción de plantas ornamentales, cuya contaminación por metales pesados no ha sido suficientemente estudiada, especialmente en lo concerniente a las aguas de riego. El objetivo de este trabajo de investigación fue determinar cuantitativamente por espectroscopía de emisión atómica mediante plasma de acoplamiento inductivo, el grado de contaminación por metales pesados de las aguas de riego de la Industria Florícola "White River Roses" del cantón Penipe. Los resultados mostraron que, en general, el agua de riego que se utiliza en esta industria agrícola no contiene cantidades significativas de metales pesados.

Palabras claves: Agua de riego, contaminación, metales pesados.

\section{A bstract}

The growth of agribusiness in Ecuador in recent years has caused serious problems of environmental contamination affecting its natural resources, so it is important to implement effective strategies to determine the sources of pollution and mitigate their impacts on the environment. One of the most important is the presence of heavy metals, therefore, it is essential to determine quantitatively them to carry out their proper management. The province of Chimborazo is located in the central zone of Ecuadorian Andes and one of its main agroindustries is related to the production of ornamental plants, whose contamination by heavy metals has not been sufficiently studied, especially with regard to the irrigation waters. The aim of this research was to determine quantitatively by atomic emission spectroscopy using inductive coupling plasma, the degree of contamination by heavy metals of the irrigation water of the Floriculture Industry White River Roses of the Penipe Canton. The results showed that, in general, the irrigation water used in this agricultural industry does not contain significant amounts of heavy metals.

Keywords: Irrigation water, pollution, heavy metals. 


\section{INTRODUCCIÓN}

El agua es un recurso natural fundamental para el cultivo de plantas florícolas. La calidad del agua de riego tiene un impacto considerable sobre el crecimiento exitoso y la productividad de las plantas $(1,2)$ y su conocimiento permite realizar los cambios necesarios para garantizar la productividad a largo plazo $(1,3)$.

Actualmente, la mayor preocupación sobre la seguridad del agua es la potencial presencia de contaminantes químicos, procedentes de fuentes industriales, agrícolas y urbanas (4). Los metales pesados son elementos químicos metálicos que tienen una densidad relativamente alta y son tóxicos o venenosos a bajas concentraciones (5), provocando problemas de toxicidad en los organismos vivos debido a que son bioacumulables (6). Elementos como níquel, cromo, cadmio, plomo, arsénico, selenio y mercurio, aún en pequeñas proporciones son potencialmente tóxicos. Una de las principales causas de la contaminación del suelo con metales pesados (plomo, cadmio, cromo, zinc, cobre, níquel, mercurio, manganeso, selenio y arsénico, entre otros) es el riego con agua de cauces y aguas residuales contaminadas $(7,8)$. En las plantas el mayor riesgo de los metales pesados radica en que pueden acumularse en las partes comestibles de los cultivos dedicados al consumo humano o para alimento de animales. La capacidad de absorción de las plantas con respecto a metales pesados es variable, lo que abre la posibilidad de adaptar la elección de cultivos según el grado y tipo de contaminación. La absorción de metales pesados en las plantas (especialmente cadmio y plomo) también varía según el $\mathrm{pH}$ del suelo (9). Las plantas ornamentales se han propuesto para crecimiento en suelos contaminados con metales pesados, y también para su uso en fitoremediación, determinándose que la producción de biomasa se reduce significativamente en los suelos enriquecidos con metales pesados que se concentran más en las raíces que en la parte aérea de las plantas (10). Estudios realizados en la industria florícola, muestran que los residuos de rosas tienen importantes cantidades de elementos potencialmente tóxicos: $\mathrm{Ni}, \mathrm{Cr}, \mathrm{Pb}, \mathrm{As}$ y Se (11). Esto indicaría que este tipo de plantas podrían utilizarse como hiperacumuladores.

La espectroscopía de emisión atómica (AES) es una técnica de análisis simultáneo multielemental que permite detectar casi todos los elementos de la tabla periódica (12). El plasma es un gas ionizado que se induce en una corriente de flujo de argón. Para las emisiones atómicas comúnmente se emplea la antorcha de plasma de acoplamiento inductivo (ICP) que actúa simultáneamente como atomizador y fuente de excitación (12). El método de Plasma de Acoplamiento Inductivo (ICP) es altamente sensible para la determinación de metales pesados que permite detectar cualitativa y cuantitativamente más de 70 elementos $(13,14)$. En las técnicas de absorción atómica, la absorción o emisión de luz se aplica a átomos aislados, lo cual evita la complejidad de los enlaces moleculares (14). Cuando se acopla el ICP con el espectrómetro de masas se obtiene un sistema de análisis multi elemental de elevadísima sensibilidad, con límites de detección, para algunos elementos, inferiores a 0,1 partes por trillón $(0,1 \mathrm{pg} / \mathrm{mL})$ y que se determinan con $\mathrm{LOD}=\mathrm{c} /\left(\mathrm{I}_{\mathrm{L}+\mathrm{T}}-\mathrm{I}_{\mathrm{T}}\right)^{*} \mathrm{I}_{\mathrm{T}} *(3 / 100)(15)$. El objetivo de esta investigación fue determinar cuantitativamente por ICP, el grado de contaminación por metales pesados de las aguas de riego de la Industria Florícola White River Roses.

\section{MATERIALES Y MÉTODOS}

\section{Procedimiento experimental}

El experimento se realizó en la Industria florícola White River Roses cantón Penipe - Ecuador $\left(1.6^{\circ} \mathrm{S}, 78.5^{\circ} \mathrm{W}\right)$. Se tomaron muestras compuestas en cuatro diferentes sitios de la cadena de irrigación: entrada de agua del río Blanco (A), entrada de agua del canal de riego (B), reservorio (C) y red de distribución (D). Se realizaron tres muestreos en diferentes fechas. El agua del río y el agua del canal ingresan al reservorio a través de tubería, por lo que en estos dos puntos se tomaron las muestras en el agua corriente. El agua del reservorio es superficial y la muestra se tomó sumergiendo el recipiente en un lugar alejado de la orilla. El agua del sistema de distribución de riego proviene del reservorio a través de tubería y su flujo es controlado mediante válvulas. El muestreo en este punto se realizó abriendo la válvula y dejando co- 
rrer el agua por 10 minutos antes de realizar la recolección de la muestra (14).

\section{Metodología}

La temperatura se determinó in situ utilizando un termómetro HANNA HI145, cuya sonda se enjuagó previamente en el agua a ser muestreada para evitar contaminar las muestras. Luego las muestras fueron selladas, etiquetadas y transportadas en refrigeración a los laboratorios de la Escuela Superior Politécnica de Chimborazo. Las muestras de cada sitio se homogeneizaron antes de realizar los análisis de laboratorio.

La muestra obtenida se filtró utilizando papel Whatman grado 1, con la finali dad de eliminar los sólidos en suspensión (16). Se determinaron parámetros físicos: temperatura, $\mathrm{pH}, \mathrm{CE}$ (conductividad eléctrica), y químicos: Ag, As, B, $\mathrm{Ba}, \mathrm{Be}, \mathrm{Ca}, \mathrm{Co}, \mathrm{Cd}, \mathrm{Cr}, \mathrm{Cu}, \mathrm{Fe}, \mathrm{K}, \mathrm{Li}$, $\mathrm{Mg}, \mathrm{Mn}, \mathrm{Mo}, \mathrm{Ni}, \mathrm{Pb}, \mathrm{Sb}, \mathrm{Se}, \mathrm{Si}, \mathrm{Sn}$, Sr, Ti, Tl, V, Zn. Para la determina ción del potencial de hidrógeno $(\mathrm{pH})$ se empleó el procedimiento de ensayo específico PEE/LABCES - TTA/05 basado en el método APHA 4500-H + B (17). Para el análisis de los metales pesados por ICP se realizó la digestión de la muestra según el procedimiento indicado por (18) con digestión ácida asistida con microondas para muestras y extractos acuosos (17). Los metales y metaloides se determi naron mediante el procedimiento de ensayo específico PEE/ LABCESTTA / 174 basado en el método EPA 200.7 (19) utilizando un espectrofotómetro Thermo Scientific iCAP 6000 con automuestreador CETAC ASX -520. Se preparó la solución patrón para cada analito diluyendo $1 \mathrm{~mL}$ de los estándares de calibración provistos por INORGANIC VENTURES $(1000 \pm 10) \mu \mathrm{g} / \mathrm{mL} 2$ $\% \mathrm{HNO} 3(\mathrm{v} / \mathrm{v})$ aforando a $100 \mathrm{~mL}$ con agua desionizada.

Durante dos horas se realizó la purga con Ar para estabilizar los componentes ópticos del equipo y se colocaron las muestras digeridas en el automuestreador desde donde pasan al ICP-AES para los análisis multielementales.

Los análisis de todas las muestras se realizaron por triplicado.

\section{Métodos estadísticos}

Los efectos significativos de las variables fueron determinados mediante análisis de varianza de una vía (ANOVA), al nivel de significancia del 5\%. Las medias de las muestras fueron separadas usando el test de Tukey-b. Todos los parámetros fueron además explorados con análisis de componentes principales (ACP) para describir las variables correlacionadas en términos de un nuevo conjunto de variables no correlacionadas, las cuales son combinaciones lineales de las variables originales. Las nuevas variables calculadas se llaman componentes principales (CPs) y son mutuamente ortogonales y no correlacionadas. Las primeras CPs, en orden descendente, explican la mayoría de la varianza total de las variables originales (20). El ACP permite representar el conjunto de datos de una manera fácil de interpretar y fue aplicado a los valores medios de las tres repeticiones de cada muestra. El análisis de los datos se realizó después de aplicar la rotación normalizada Varimax al sistema coordinado de las CPs. Las cargas del factor (loadings) $>|0.6|$ indican correlaciones significativas entre las variables originales y las componentes $(21,22)$. En base a los resultados del $\mathrm{ACP}$, una clasificación jerárquica (usando la distancia Euclidiana y la regla de aglomeración de Ward) fue corrida sobre la matriz de datos para identificar similaridades entre las muestras. Se obtuvo un dendograma que clasifica las muestras. Finalmente, se hizo una clasificación no jerárquica usando el algoritmo k-means para identificar las muestras homogéneas y las variables más relevantes asociadas a cada muestra. El número óptimo de grupos se ha seleccionado con el criterio del agrupamiento cúbico (22). Los análisis estadísticos fueron realizados con el software estadístico SPSS v. 22.0.

\section{RESULTADOS Y DISCUSIÓN}

\section{Parámetros físicos}

La temperatura promedio de todo el sistema de riego fue de $13.2{ }^{\circ} \mathrm{C}$. Las aguas más frías provienen del canal de riego y del río con $9.90^{\circ} \mathrm{C}$ y $11.07{ }^{\circ} \mathrm{C}$, respectivamente. El reservorio y la red de distribución tuvieron temperaturas similares con $15.97{ }^{\circ} \mathrm{C}$ y $15.83{ }^{\circ} \mathrm{C}$, respectivamente. En el agua analizada se encontraron valores de $\mathrm{pH}$ lige- 


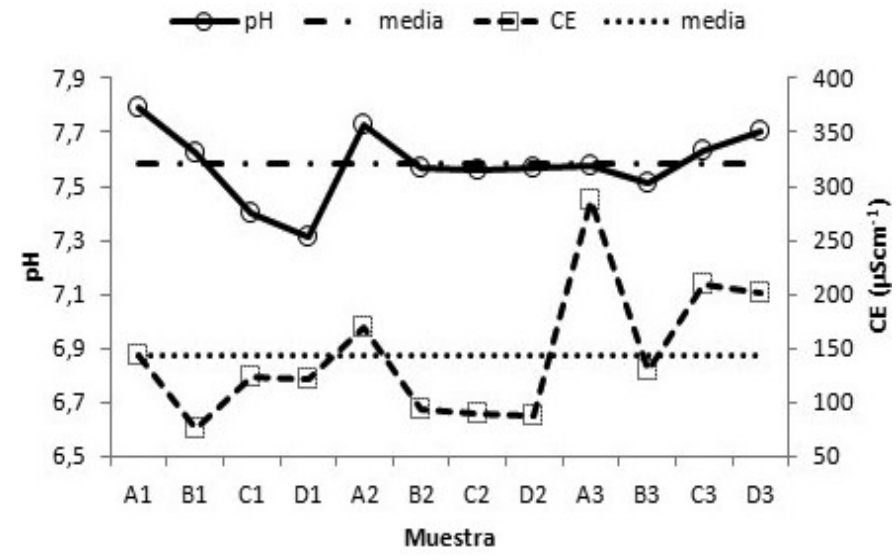

Figura 1. Conductividad eléctrica y $\mathrm{pH}$ del agua

ramente alcalinos, lo cual indica que los metales pesados están menos disponibles. El pH de la solución tiene un efecto sobre la biodisponibilidad de la mayoría de los metales pesados porque afecta el equilibrio entre la solubilidad, adsorción e intercambio de iones en el suelo $(23,24)$. $\mathrm{Al}$ aumentar el $\mathrm{pH}$, los metales son removidos de la solución del suelo y adsorbidos por los coloides disminuyendo su biodisponibilidad $(23,25)$. Todos los valores de pH están dentro del rango de límites permisibles $(6-9)$; mientras que la conductividad eléctrica mostró todos sus valores por debajo del límite máximo permitido de 700 $\mu \mathrm{Scm}^{-1}$ (figura 1). Según estos dos parámetros, el agua analizada es recomendable para el riego agrícola de conformidad con los límites establecidos por la normativa ecuatoriana sobre criterios de calidad admisibles para aguas de uso agrícola (26).

\section{Parámetros químicos}

Se analizaron 27 parámetros, pero únicamente 17 dieron resultados por encima de los límites de detección.

El agua del río tiene los promedios más altos de Fe, Mn, Mo, Sn y Ti, debido al arrastre de minerales. En el agua del canal se encuentran los valores promedio más elevados de Ag y Ba provenientes de las fuentes que lo alimentan. En el reservorio se han encontrado los niveles más altos de $\mathrm{Ca}, \mathrm{Cd}, \mathrm{K}, \mathrm{Mg}, \mathrm{Ni}, \mathrm{Pb}$, $\mathrm{Si}, \mathrm{Sr}$ y Tl; que podrían asociarse con la concentración de sus sales minerales.

Todos los analitos se encuentran dentro de los LMP establecidos por la normativa ecuatoriana sobre criterios de calidad admisibles para aguas de uso agrícola, excepto el Mo que en la entrada del agua del río presenta un promedio de 34,48

\begin{tabular}{|c|c|c|c|c|c|}
\hline Analito [ppb] & Río & Canal & Reservorio & Distribución & ANOVA \\
\hline $\mathrm{Ag}$ & $7,128 \mathrm{a}$ & $7,619 \mathrm{a}$ & $7,367 \mathrm{a}$ & $6,826 \mathrm{a}$ & NS \\
\hline $\mathrm{As}$ & $3,187 \mathrm{a}$ & $2,900 \mathrm{a}$ & $2,302 \mathrm{a}$ & $5,342 \mathrm{a}$ & NS \\
\hline $\mathrm{Ba}$ & $29,08 \mathrm{a}$ & $38,99 \mathrm{~b}$ & $30,70 \mathrm{a}$ & $20,07 \mathrm{c}$ & $* * *$ \\
\hline $\mathrm{Ca}$ & $24973 \mathrm{a}$ & $22387 \mathrm{~b}$ & $26847 \mathrm{c}$ & $20607 \mathrm{~d}$ & $*$ \\
\hline $\mathrm{Cd}$ & $2,519 \mathrm{ab}$ & $2,776 \mathrm{a}$ & $4,810 \mathrm{c}$ & $1,779 \mathrm{~b}$ & $* * *$ \\
\hline $\mathrm{Fe}$ & $742,4 \mathrm{a}$ & $165,0 \mathrm{~b}$ & $314,7 \mathrm{c}$ & $236,2 \mathrm{~d}$ & $*$ \\
\hline $\mathrm{K}$ & $1734 \mathrm{a}$ & $1013 \mathrm{~b}$ & $1817 \mathrm{c}$ & $1299 \mathrm{~d}$ & $*$ \\
\hline $\mathrm{Mg}$ & $7734 \mathrm{a}$ & $3706 \mathrm{~b}$ & $8076 \mathrm{a}$ & $5879 \mathrm{c}$ & $*$ \\
\hline $\mathrm{Mn}$ & $17,96 \mathrm{a}$ & $8,92 \mathrm{~b}$ & $12,49 \mathrm{c}$ & $9,94 \mathrm{~b}$ & $*$ \\
\hline $\mathrm{Mo}$ & $34,48 \mathrm{a}$ & $4,25 \mathrm{~b}$ & $3,21 \mathrm{~b}$ & $6,54 \mathrm{~b}$ & $*$ \\
\hline $\mathrm{Ni}$ & $4,136 \mathrm{a}$ & $3,794 \mathrm{a}$ & $4,565 \mathrm{a}$ & $0,000 \mathrm{~b}$ & $*$ \\
\hline $\mathrm{Pb}$ & $6,479 \mathrm{a}$ & $6,959 \mathrm{a}$ & $7,261 \mathrm{a}$ & $6,086 \mathrm{a}$ & $*$ \\
\hline $\mathrm{Si}$ & $9341 \mathrm{a}$ & $8496 \mathrm{~b}$ & $9662 \mathrm{c}$ & $7000 \mathrm{~d}$ & $*$ \\
\hline $\mathrm{Sn}$ & $3,214 \mathrm{a}$ & $1,813 \mathrm{a}$ & $2,454 \mathrm{a}$ & $1,557 \mathrm{a}$ & $*$ \\
\hline $\mathrm{Sr}$ & $130,4 \mathrm{a}$ & $75,1 \mathrm{~b}$ & $138,2 \mathrm{c}$ & $99,7 \mathrm{~d}$ & $*$ \\
\hline $\mathrm{Ti}$ & $23,86 \mathrm{a}$ & $2,99 \mathrm{~b}$ & $10,40 \mathrm{c}$ & $9,77 \mathrm{c}$ & $*$ \\
\hline $\mathrm{Tl}$ & $14,02 \mathrm{a}$ & $12,22 \mathrm{~b}$ & $14,17 \mathrm{a}$ & $13,28 \mathrm{a}$ & $*$ \\
\hline
\end{tabular}

***: Significativa a $\mathrm{P}<0.001$; **: Significativa a $\mathrm{P}<0.01$. *: Significativa a $\mathrm{P}<0.05$. NS: No significativa. Los valores medios en las filas seguidos por la misma letra no difieren significativamente a $\mathrm{P}<0.05$ (test Tukey-b). 


\begin{tabular}{|c|c|c|c|c|c|c|c|c|}
\cline { 2 - 9 } \multicolumn{1}{c|}{} & $\mathbf{C a}$ & $\mathbf{F e}$ & $\mathbf{K}$ & $\mathbf{M g}$ & $\mathbf{M n}$ & $\mathbf{M o}$ & $\mathbf{N i}$ & $\mathbf{S r}$ \\
\hline $\mathrm{Mg}$ & 0,715 & 0,653 & $0,985^{* * *}$ & & & & & \\
\hline $\mathrm{Mn}$ & 0,562 & $0,978^{* * *}$ & 0,759 & 0,741 & & & & \\
\hline $\mathrm{Mo}$ & 0,214 & $0,951^{* * *}$ & 0,431 & 0,427 & $0,902^{* * *}$ & & & \\
\hline $\mathrm{Si}$ & $0,933^{* * *}$ & 0,488 & 0,654 & 0,544 & 0,582 & 0,308 & $0,945^{* * *}$ & \\
\hline $\mathrm{Sr}$ & 0,784 & 0,645 & $0,997^{* * *}$ & $0,993^{* * *}$ & 0,742 & 0,409 & 0,381 & \\
\hline $\mathrm{Ti}$ & 0,396 & $0,977^{* * *}$ & 0,713 & 0,725 & $0,957^{* * *}$ & $0,917^{* * *}$ & 0,190 & 0,700 \\
\hline $\mathrm{Tl}$ & 0,675 & 0,571 & $0,877^{* * *}$ & $0,906^{* * *}$ & 0,634 & 0,363 & 0,185 & $0,891^{* * *}$ \\
\hline
\end{tabular}

***: Significativa a $\mathrm{P}<0.001$;

Tabla 2. Correlación de variables

$\mu \mathrm{g} / \mathrm{L}$ muy por encima del $\mathrm{LMP}=0,01$ $\mathrm{mg} / \mathrm{L}$ (26). El principal problema del riego con agua que contiene elevadas cantidades de molibdeno es que éste es absorbido y concentrado por las plantas. Altas concentraciones de molibdeno rara vez retrasan el crecimiento de la planta, pero pueden disminuir la disponibilidad de cobre provocando hipocruposis (27) y síndrome de molibdenosis en el organismo de los animales rumiantes (28). La concentración elevada de Mo puede deberse a que en las tierras altas existen minerales que lo contienen y que son transportados por el caudal del río.

Con excepción de $\mathrm{Ag}$, As y $\mathrm{Pb}$, existen diferencias significativas en la concentración media de los metales de acuerdo al sitio de muestreo.

La tabla 2 muestra las correlaciones entre los elementos. Dado que las correlaciones son positivas, significa que el incremento de la concentración de un analito implica el aumento de la concentración del otro. La mejor de todas las correlaciones es para K-Sr con $\mathrm{r}=0.997$. Los elementos que están correlacionados aportan la misma información, por lo que podría desecharse uno de ellos, reduciendo de esta manera el número de metales en estudio.

\section{Análisis multivariado}

En el análisis de componentes principales la medida de Kaiser-Meyer-Olkin de suficiencia del muestreo (KMO) fue mayor que 0.5 y el test de esfericidad de Bartlett mostró un valor $\mathrm{P}<0.001$. Además, ninguna de las variables presentó un valor de extracción $<0.5$. Por tanto, el modelo establecido fue adecuado. Las dos componentes principales (CP) explicaron $87.44 \%$ de la variabilidad, con contribuciones CP1: $53.68 \%$ y CP2: $33.76 \%$. CP1 estuvo constituida por Mn, Ti, Mg, Tl, K, Fe, Sr, Sn y Mo. Las demás variables constituyeron $\mathrm{CP} 2$.

Todas las variables en CP1 están positivamente correlacionadas, mientras que en $\mathrm{CP} 2$ el As está correlacionado negativamente con las demás variables.

\begin{tabular}{|c|c|c|}
\hline & CP1 & CP2 \\
\hline Varianza explicada (\%) & 53,68 & 33,76 \\
\hline Varianza acumulativa (\%) & 53,68 & 87,44 \\
\hline $\mathrm{Mn}$ & 0,947 & \\
\hline $\mathrm{Ti}$ & 0,943 & \\
\hline $\mathrm{Mg}$ & 0,913 & \\
\hline $\mathrm{Tl}$ & 0,907 & \\
\hline $\mathrm{K}$ & 0,907 & \\
\hline $\mathrm{Fe}$ & 0,905 & \\
\hline $\mathrm{Sr}$ & 0,900 & \\
\hline Sn & 0,879 & \\
\hline Mo & 0,763 & \\
\hline As & & $-0,978$ \\
\hline $\mathrm{Pb}$ & & 0,977 \\
\hline $\mathrm{Ni}$ & & 0,917 \\
\hline $\mathrm{Ag}$ & & 0,882 \\
\hline $\mathrm{Cd}$ & & 0,843 \\
\hline $\mathrm{Si}$ & & 0,843 \\
\hline $\mathrm{Ba}$ & & 0,817 \\
\hline $\mathrm{Ca}$ & & 0,755 \\
\hline
\end{tabular}

Tabla 3. Loadings de las variables para las componentes principales (CP) extraídas mediante análisis ACP, para las muestras estudiadas (sólo se reportan los loadings significativos $>|0.6|$ ). 


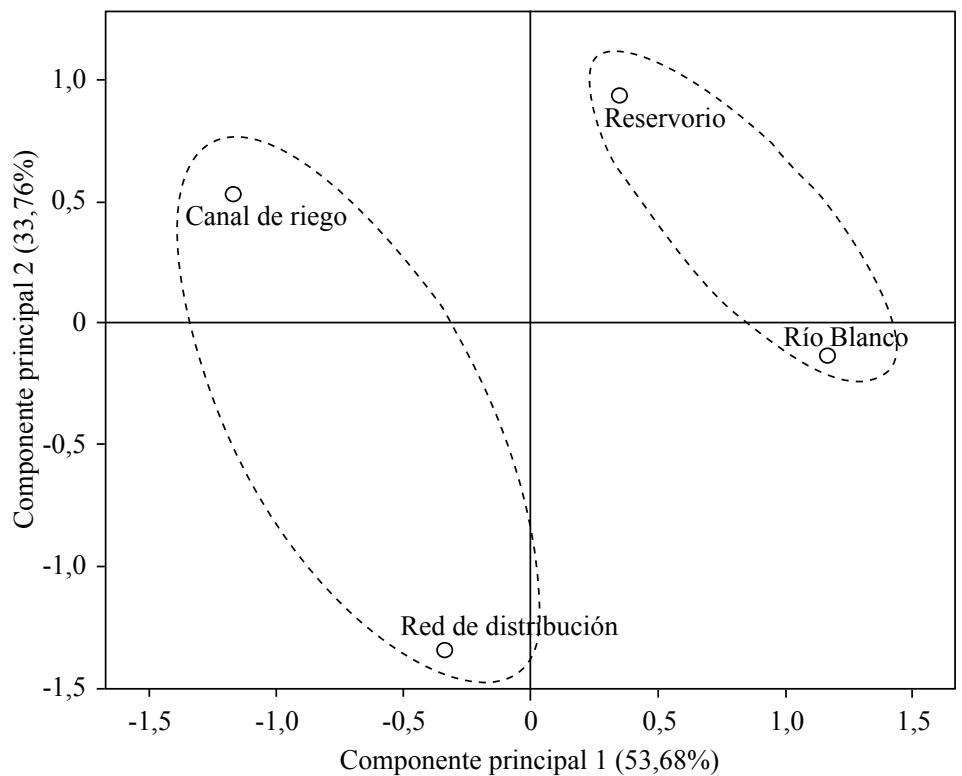

Figura 2. Puntajes de las muestras en el análisis de componentes principales

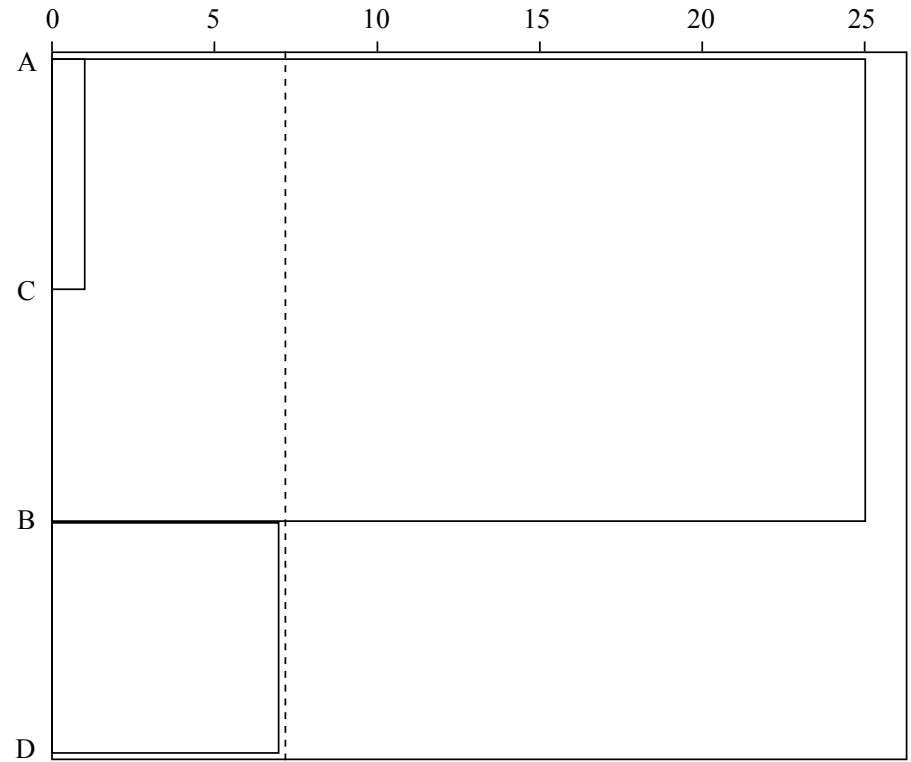

Figura 3. Agrupación jerárquica de las muestras probadas en el presente estudio.

\begin{tabular}{|l|c|c|c|}
\hline \multicolumn{1}{|c|}{ Grupo 1 } & Distancia & Grupo 2 & Distancia \\
\hline Río Blanco & 2,207 & Canal de riego & 2,658 \\
\hline Reservorio & 2,207 & Red de distribución & 2,658 \\
\hline
\end{tabular}

Tabla 4. Grupos k-means
Las muestras provenientes del río Blanco y del reservorio se distribuyeron sobre el lado positivo de CP1. Sobre el lado negativo se encuentraron las muestras que provienen del canal de riego y de la red de distribución.

El análisis de agrupación jerárquica se usó para estudiar la similaridad entre las muestras. Usando un corte de 7.2 en la distancia normalizada, se identificaron dos grupos : un grupo que consistieron de las muestras provenientes del río Blanco y del reservorio, y otro grupo compuesto por las muestras del canal de riego y de la red de distribución.

El análisis de agrupación no jerárquico (k-means) dio como resultado dos grupos: un grupo compuesto por las muestras provenientes del río Blanco y del reservorio, y otro grupo compuesto por las muestras del canal de riego y de la red de distribución.

Los grupos obtenidos por los métodos de agrupación jerárquica y no jerárquica, corroboraron completamente los resultados obtenidos por el ACP - Las muestras de agua del río Blanco y del reservorio pertenecen al mismo grupo debido a que el reservorio es alimentado en mayor volumen por el agua del río.

\section{CONCLUSIONES}

Los parámetros $\mathrm{pH}$ y $\mathrm{CE}$ se encuentra dentro de los rangos establecidos en la legislación ecuatoriana, por tanto el agua es apropiada para el riego agrícola. El agua es apta para su uso en irrigación agrícola, dado que las concentraciones de los metales estudiados se encuentran dentro de los límites máximos permisibles, excepto por el Mo proveniente del agua del río. Aunque las cantidades de metales pesados y otras sustancias tóxicas son del orden de partes por billón, éstas producen la contaminación del suelo por acumulación debido al riego frecuente. El análisis multivariado determinó que las muestras de agua del río y del reservorio pertenecen al mismo grupo. 


\begin{tabular}{|c|c|c|c|c|c|}
\hline & \multicolumn{2}{|c|}{ Grupo 1} & \multicolumn{2}{|c|}{ Grupo 2} & \multirow[t]{2}{*}{ ANOVA } \\
\hline & Media & $\mathrm{DE}$ & Media & $\mathrm{DE}$ & \\
\hline $\mathrm{Ag}$ & 7,25 & 0,17 & 7,22 & 0,56 & NS \\
\hline As & 2,74 & 0,63 & 4,12 & 1,73 & NS \\
\hline $\mathrm{Ba}$ & 29,89 & 1,15 & 29,53 & 13,38 & NS \\
\hline $\mathrm{Ca}$ & 25910,00 & 1324,65 & 21496,67 & 1258,65 & NS \\
\hline $\mathrm{Cd}$ & 3,66 & 1,62 & 2,28 & 0,70 & NS \\
\hline $\mathrm{Fe}$ & 528,53 & 302,45 & 200,60 & 50,39 & NS \\
\hline $\mathrm{K}$ & 1775,17 & 58,69 & 1155,78 & 202,54 & NS \\
\hline $\mathrm{Mg}$ & 7905,00 & 241,36 & 4792,50 & 1536,54 & NS \\
\hline $\mathrm{Mn}$ & 15,23 & 3,87 & 9,43 & 0,72 & NS \\
\hline Mo & 18,85 & 22,11 & 5,40 & 1,62 & NS \\
\hline $\mathrm{Ni}$ & 4,35 & 0,30 & 1,90 & 2,68 & NS \\
\hline $\mathrm{Pb}$ & 6,87 & 0,55 & 6,52 & 0,62 & NS \\
\hline $\mathrm{Si}$ & 9501,33 & 226,75 & 7747,67 & 1057,83 & NS \\
\hline $\mathrm{Sn}$ & 2,83 & 0,54 & 1,69 & 0,18 & NS \\
\hline $\mathrm{Sr}$ & 134,30 & 5,47 & 87,37 & 17,41 & NS \\
\hline $\mathrm{Ti}$ & 17,13 & 9,52 & 6,38 & 4,79 & NS \\
\hline $\mathrm{Tl}$ & 14,10 & 0,11 & 12,75 & 0,75 & NS \\
\hline
\end{tabular}

$* * *$ : Significativa a $\mathrm{P}<0.001$;

Tabla 5. Valor medio y desviación estándar de las variables observadas mediante agrupación k-means.

\section{AGRADECIMIENTOS}

Los autores expresan su agradecimiento a la Empresa White River Roses por las facilidades brindadas en la recolección de las muestras para esta investigación y a la Escuela Superior Politécnica de Chimborazo por el apoyo logístico y académico.

1. Bauder TA, Waskom RM, Sutherland PL, Davis JG. Irrigation Water Quality Criteria. [cited 2017 Aug 1]; Available from: https://dspace.library.colostate.edu/bitstream/handle/10217/182905/AEXT_ ucsu2062205062011.pdf?sequence $=1$

2. Yohannes DF, Ritsema CJ, Solomon H, Froebrich J, van Dam JC. Irrigation water management: Farmers' practices, perceptions and adaptations at Gumselassa irrigation scheme, North Ethiopia. Agric Water Manag [Internet]. 2017;191:16-28. Available from: http:/www.sciencedirect.com/science/article/pii/ S0378377417301865

3. Levin M, Gealt M. Biotratamiento de residuos tóxicos y peligrosos. Madrid: McGraw-Hill; 1997.

4. Bautista Zúñiga F. Introducción al estudio de la contaminación del suelo por metales pesados. Ediciones de la Universidad Autónoma de Yucatán; 1999. 109 p.

5. Restrepo Acevedo AM. Calibración y validación del muestreador pasivo seleccionado, para la determinación de mercurio y metales pesados, simulando las condiciones ambientales reales en el laboratorio. Corporación Universitaria Lasallista; 2015.

6. Guzmán A, Sánchez S, Flores D, Quevedo N, Váldes R. La Biorremediación, un método para descontaminar áreas agrícolas. Anu DICT-UNAH, Cuba 51pp, formato electrónico. 2005; 
7. Orroño D, Lavado R. Heavy metal accumulation in Pelargonium hortorum: Effects on growth and development. Phyt (Buenos Aires) [Internet]. 2009 [cited 2017 Aug 1];75. Available from: http://www. scielo.org.ar/img/revistas/phyton/v78n1/html/v78n1a13.htm

8. Wu Q, Leung JYS, Geng X, Chen S, Huang X, Li H, et al. Heavy metal contamination of soil and water in the vicinity of an abandoned e-waste recycling site: Implications for dissemination of heavy metals. Sci Total Environ [Internet]. 2015;506:217-25. Available from: http://www.sciencedirect.com/science/ article/pii/S0048969714015836

9. Gavilanes-Terán I, Paredes C, Pérez-Espinosa A, Ángeles Bustamante M, Gálvez-Sola L, Jara-Samaniego J. Opportunities and Challenges of Organic Waste Management from the Agroindustrial Sector in South America: Chimborazo Province Case Study. Commun Soil Sci Plant Anal [Internet]. 2014;46(sup1):13756. Available from: http://www.scopus.com/inward/record.url?eid=2-s2.0-84924965289\&partnerID=tZOtx3y1

10. Vilanova Gisbert E, Sogorb Sánchez MA. Técnicas analíticas de contaminantes químicos: aplicaciones toxicológicas, medioambientales y alimentarias. Ediciones Díaz de Santos; 2004.

11. Skoog DA. Fundamentals of analytical chemistry. Grupo Editorial Norma; 2004.

12. Sogorb Sánchez MA, Vilanova Gisbert E. Técnicas analíticas de contaminantes químicos : aplicaciones toxicológicas, medioambientales y alimentarias. Ediciones Díaz de Santos; 2004. 305 p.

13. Marrugo-Negrete JL, Urango-Cardenas ID, Núñez SMB, Díez S. Atmospheric deposition of heavy metals in the mining area of the San Jorge river basin, Colombia. Air Qual Atmos Heal. 2014;7(4):577-88. 14. Mulki M, Yánez O, Jaramillo M, Jácome A, Leiva E, Jaramillo P. Manual para muestreo de aguas y sedimentos. Quito; 1998.

15. Feist B, Mikula B. Preconcentration of heavy metals on activated carbon and their determination in fruits by inductively coupled plasma optical emission spectrometry. Food Chem [Internet]. 2014 [cited 2018 Mar 25];147:302-6. Available from: https ://www sciencedirect .com/science /article /pii/S 0308814613014349

16. Amin N-, Hussain A, Alamzeb S, Begum S. Accumulation of heavy metals in edible parts of vegetables irrigated with waste water and their daily intake to adults and children, District Mardan, Pakistan. Food Chem [Internet]. 2013;136(3):1515-23. Available from: http://www.sciencedirect.com/science/article/pii/S0308814612014628

17. American Public Health Association, APHA. Water Environment Federation (1992) Standard methods for the examination of water and wastewater. 1996;

18. Islam M, Ahmed M, ... MR-E, 2015 undefined. Heavy metal pollution in surface water and sediment: a preliminary assessment of an urban river in a developing country. Elsevier [Internet]. [cited $2018 \mathrm{Mar}$ 25]; Available from: https://www.sciencedirect.com/science/article/pii/S1470160X14003719

19. US EPA O. Method 200.7: Determination of Metals and Trace Elements in Water and Wastes by Inductively Coupled Plasma-Atomic Emission Spectrometry. [cited 2018 Mar 24]; Available from: https:// www.epa.gov/homeland-security-research/method-2007-determination-metals-and-trace-elements-water-and-wastes

20. Gil MV, Calvo LF, Blanco D, Sánchez ME. Assessing the agronomic and environmental effects of the application of cattle manure compost on soil by multivariate methods. Bioresour Technol [Internet]. 2008 Sep [cited 2018 Mar 3];99(13):5763-72. Available from: http://linkinghub.elsevier.com/retrieve/pii/ S0960852407008528

21. Paredes C, Medina E, Bustamante MA, Moral R. Effects of spent mushroom substrates and inorganic fertilizer on the characteristics of a calcareous clayey-loam soil and lettuce production. Soil Use Manag. 2016;32(4):487-94.

22. Rinaldi S, De Lucia B, Salvati L, Rea E. Understanding complexity in the response of ornamental rosemary to different substrates: A multivariate analysis. Sci Hortic (Amsterdam) [Internet]. 2014; 176(September):218-24. Available from: http://dx.doi.org/10.1016/j.scienta.2014.07.011 
23. Torres G, Navarro EA, Languasco J, Campos K, Cuizano N. Estudio preliminar de la fitoremediación de cobre divalente mediante Pistia stratioides (lechuga de agua). Descargado http//cienciaescolar net/proyectos/wpcontent/uploads/2009/09/Art_14_Torres_lechuga_agua pdf el. 2007;15.

24. Meng W, Wang Z, Hu B, Wang Z, Li H, Goodman RC. Heavy metals in soil and plants after long-term sewage irrigation at Tianjin China: A case study assessment. Agric Water Manag [Internet]. 2016;171:15361. Available from: http://www.sciencedirect.com/science/article/pii/S037837741630097X

25. Gaete H, Aránguiz F, Cienfuegos G, Tejos M. Metales pesados y toxicidad de aguas del río Aconcagua en Chile. Quim Nova. 2007;30(4):885.

26. Ministerio del Ambiente. Anexo 1 Del Libro Vi Del Texto Unificado De Legislacion Secundaria Del Ministerio Del Ambiente: Norma De Calidad Ambiental Y De Descarga De Efluentes A1 Recurso Agua. Norma de Calidad Ambiental y de Descarga de Efluentes : Recurso Agua. 2014.

27. Ward GM. Molybdenum toxicity and hypocuprosis in ruminants: a review. JAnim Sci. 1978;46(4):107885.

28. Igarza LM, Auza N Buenos Aires (Argentina). Fac. de Ciencias Veterinarias) Y (Universidad N del C. Molybdenum in ruminants: a review of the physiological and toxic aspects. Vol. v. 27, Archivos de Medicina Veterinaria (Chile). 1995. 